\title{
Research on software development method of SOA Architecture
}

\author{
Peichun Suo \\ Yunnan College of Business Management, 650304, China
}

Keywords: SOA Architecture, Software Development, Engineering Construction, CMI.

\begin{abstract}
With the advent of the information age, software development has been paid attention to by the society. SOA architecture is a common structure in software development process, which can reduce development cost, realize loose coupling structure and meet the needs of various software function development. This paper will study three aspects of SOA architecture software development technology analysis, practice technology and case analysis, aiming at promoting software development and construction in China.
\end{abstract}

\section{Introduction}

Software is an important way to improve platform performance and execute commands by default. software development method, can affect the platform construction effect. With the further development of software development, commercial system functions, platform construction work is becoming more and more complex. In the process of traditional platform construction, the software development and construction method of tight coupling structure is used, but this construction method also makes the information isolated, which is not conducive to the exchange of information among the parties[1]. SOA architecture can reuse existing modules, speed up software development and improve platform performance by building a distributed system. SOA architecture is both an architectural model and a programming model, which is of great significance to project construction. In view of this, it is necessary to study SOA architecture software.

\section{SOA Architecture Software Development of Technical Analysis}

SOA architecture software has different definitions of directional differences based on the perspective of observation. (1) Defining SOA architecture software from a structural perspective belongs to loosely coupled software, and each part is independent and can be reused to form a service system. The SOA architecture software service system will announce the interface to the outside world, and has the clear meaning, can support the software development work to carry on. Figure 1: A schematic diagram of the software development method structure of the SOA architecture, including three parts: Services Registry, Services Consumer, and Services Provider, with three functions: Find, Publish, and Bind Invoke.

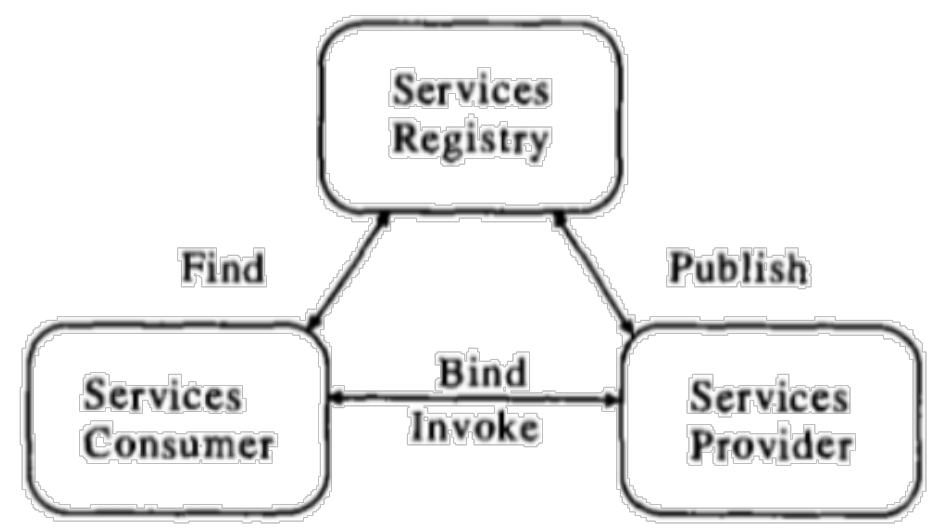

Figure 1 Software development methods for SOA architecture structure diagram

There are three roles involved in the public service architecture: service consumers, service 
providers and service registry.

First, the service consumer: in this participation process needs to serve the consumer or the application program, the concrete step is:(1) uses the registry central service inquiry function to inquire the information;(2) according to the interface explanation information, uses the service binding function and the transmission protocol function to provide the service.

Second, service providers: common service entities according to actual needs, able to accept, process remote service requests, specific steps:(1) provide services at service consumers; and (2) provide access services to service providers based on information (interfaces) published by the service entity center.

Third, the service registry: its main function is to provide the display for the center location, the specific steps are:(1) to provide the inquiry information service for the consumer;(2) to provide the target interest interface information for the service consumer by storing the information base.

Typically, the entity roles between the service consumers, providers, and registry are fixed, enabling multiple functions at the same time to build a service architecture. The service architecture generally has three functions: publishing, discovering, calling, and binding. Publishing refers to being able to describe the service information in the process of being accessed by the service consumer to facilitate its implementation call and discovery. Found, refers to the query registration function to achieve the service requester center location function requirements. Calling and binding means that after obtaining the service information, and based on the implementation of the call and binding service, and closely related to the release, discovery link.

In SOA architecture software, service is the most basic part of it, belonging to the underlying technology and the abstract infrastructure in business requirements. The development of structural software needs to follow three steps: first, to clarify the dynamic relationship. The development process needs to make clear the specific relationship between the available service and the functional requirements, and the dynamic development trend, so that the underlying technology of the service; second, the definition is clear. SOA architecture software The basic service items in the system need clear function and clear definition requirements, and the function should be relatively single, in which the independent deployment service in the system structure cannot depend on the independent existence of other systems; third, the realization of functional service requirements, through the process to meet the service function.

From the above analysis, we can know that SOA architecture software Compared with the traditional development method, it has obvious structural features such as loose coupling of structure and sharing service. The advantages are as follows:

First, it is easy to integrate. SOA architecture software can transform existing systems and applications into new service functions without modifying the system. Access to legacy systems with SOA encapsulation to avoid modifying existing system structures.

Second, the standard framework. SOA architectures can carry a variety of groups and can be well integrated into a structured system as long as the components meet the relevant standards. In the software development process, different card development groups can be added to the infrastructure framework.

Third, improve efficiency. SOA systems are highly reusable and make the most of existing services in the system. For example, the original service items in the system can be used in the design and development, testing and deployment process, which greatly shortens the service cycle.

Fourth, reduce costs. Software development is a high consumption cost project, using SOA system can effectively reduce secondary development costs, and still maintain the loose coupling characteristics of the system.

\section{SOA Architecture Software Implementation Technology}

The implementation of SOA architecture software mainly relies on Web services to enable users to use interfaces to access services. In theory, users and potential users can use Web services to implement interface calls, adapting to the powerful and flexible service requirements of the SOA architecture. 
Through the structural analysis, we can know that the SOA architecture software structure is loose and not restricted by the specific technology system, and belongs to the specific technology collection system, such as combining RMI, web services, com, etc. In a broad sense, SOA architecture software can be implemented by using the above technology, but in practice, it can only meet the requirements of SOA architecture software technology by using the conforming gravity technology[2]. In the previous software development process, distributed technology was adopted, and the SOA architecture software with Web service technology as its core was more reliable. In general, Web technology is not considered as the necessary component condition to implement the software development of SOA architecture, but because of its obvious advantage, it has an important position in SOA, and it is also the preferred object in software implementation technology.

\subsection{Critical Technology Analysis}

SOAP, WSDL, UDDI make up the Web service stack (as shown in Table 1) and are key technologies for the implementation of Web service skills. SOAP primarily defines the network delivery XML message format and is based on XML as a communication protocol. The SOAP structure is "SOAP envelope + SOAP header element (surrounded by envelope elements)+ SOAP body elements ". The WSDL is run in XML language and can provide other programs with methods, usage rules, etc. UDDI can define the format with XML as the main service object [3].

Table 1 SOAP, WSDL, UDDI constitute web service stack

\begin{tabular}{|c|c|}
\hline Projects & Role \\
\hline UDDI & $\begin{array}{c}\text { Publication and discovery of clothing } \\
\text { paintings }\end{array}$ \\
\hline WSDL & Service description \\
\hline SOAP & XML message \\
\hline HTTP. SMTP. FTP. etc & Network transmission \\
\hline
\end{tabular}

\subsection{Service Runtime}

There are many similarities between SOA architecture and Web services, especially in running services, which are designed to publish WSDL service information through UDDI. During use, the servicer can obtain the relevant information by means of registration query, the following steps are as follows:(1) from the registry to query the WSDL document information required by the servicer user;(2) from the document to obtain the interactive information;(3) to provide SOAP request information; and (4) the service to provide the corresponding response information.

\section{Case Study}

\subsection{Subjects}

Research object: the selected computer teaching management system is analyzed, and the system is analyzed according to the CELTS-20.

The functional requirements for operationalization are mainly four points: first, to implement the same curriculum in different systems; second, each independently developed curriculum has a set of characteristics, can become a curriculum system; third, the curriculum can be transferred to achieve the system transfer function; fourth, to be able to make students more convenient analysis of data. According to the principle of SOA architecture software development, it is necessary to ensure that the modules in the system can be shared with different platforms in a variety of environments.

Reuse refers to reuse, which is also the core concept in the process of SOA system construction, and it is necessary to use the original service to build the information system as much as possible in the process of software development, so as to reduce the cost of project development. Considering the need to obtain student information in the educational administration system, it is necessary to set up corresponding functions to construct multiple systems with this function simultaneously through 
reuse[4].

\subsection{Platform Design}

Java platform can be used in the design of the system, and it is easy to build a SOA system with educational administration function. After analyzing, J2EE can basically meet the requirement of this hypothesis, and the concrete construction system is shown in figure 2.

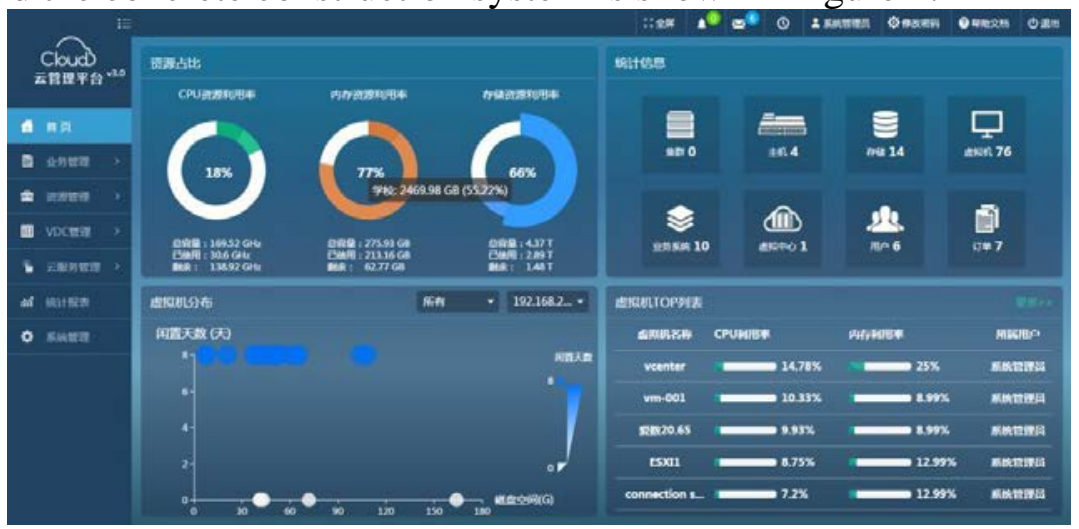

Figure 2 Computer teaching management system

\subsection{System Implementation Function}

In the educational administration system, students need to register multiple systems to meet the information login, access, management needs. So in the process of creating a Wen service, the designer can use WSAD to create a service and generate a WSDL file with Bean.

\section{Conclusion}

SOA architecture software development, greatly enhance the platform's functionality and development, with its loosely coupled structure to give software development more innovative, help to develop new ideas. At the same time, software development under SOA system can use some independent functions repeatedly, and can reduce development cost. In this paper, an example of the development of SOA architecture software is analyzed based on the computer teaching management system.

\section{References}

[1] Wu, Wenqing., Xiu, Yahui. Research on software development methods based on SOA architecture. Vocational Technology, vol. 17, no. 10, pp. 94-96, 2018.

[2] Yang, Guojun. Research on software development methods based on SOA architecture. Communication World, no. 6, pp. 267-268, 2018.

[3] Yun, Xiaolong. Research on software development methods based on SOA architecture. Information Systems Engineering, no. 11, pp. 62, 2017.

[4] Ouyang, answered. Research on software development methods based on SOA architecture. Computer Fans, no. 20, pp. 201, 2017. 